\title{
A novel method for the synthesis of $\mathrm{N}$-sulfonyl aldimines using $\mathrm{AlCl}_{3}$ under solvent-free conditions (SFC)
}

\author{
Hashem Sharghi*, Mona Hosseini-Sarvari,* and Sakineh Ebrahimpourmoghaddam \\ Department of Chemistry, College of Science, Shiraz University, Shiraz 71454, I. R. Iran \\ E-mail: shashem@susc.ac.ir, hossaini@susc.ac.ir
}

\begin{abstract}
$\mathrm{N}-$ Sulfonyl aldimines are powerful synthetic intermediates in organic synthesis and industrial application. They are prepared expeditiously under solvent-free conditions (SFC) by reaction between different aromatic aldehydes and sulfonamides in the presence of $\mathrm{AlCl}_{3}$ in good to excellent yields.
\end{abstract}

Keywords: N-sulfonyl aldimine, sulfonamide, SFC: solvent-free conditions, $\mathrm{AlCl}_{3}$

\section{Introduction}

N-Sulfonyl aldimines have proven to be useful intermediates in organic synthesis. ${ }^{1}$ They have been increasing in importance because they are one of the few types of electron deficient imines that are stable enough to be isolated but reactive enough to undergo addition reaction. ${ }^{2}$ They find elegant application in inverse electron demand Diels-Alder chemistry ${ }^{3-8}$, and ene ${ }^{9,10}$, addition ${ }^{11}$, and radical reactions. ${ }^{12}$ They are also valuable precursors for preparation of optically active 2imidazolines $^{13}$, aziridines ${ }^{14,15}$ and $\mathrm{N}$-sulfonyloxaziridines which have utility as chiral oxidants. ${ }^{16}$ Several methods for the preparation of $\mathrm{N}$-sulfonylimines have appeared in the literature. Lichtenburger and Kretar first prepared such compounds by a Lewis acid catalyzed direct condensation $^{17}$, Jennings and Lovely used titanium tetrachloride and triethylamine for the synthesis of $\mathrm{N}$-tosylimines ${ }^{18}$, rearrangements of sulfinate esters of oximes have also served as a convenient approach, Kresze pioneered an imine-transfer reaction with aromatic aldehydes utilizing sulfinylsulfonamides. ${ }^{19,}{ }^{20} N, N^{\prime}$-Bis(aryl-sulfonylthio)- and selenodiimides ${ }^{21}$ have also been effective but suffer from lack of chemoselectivity since they undergo Diels-Alder and ene reactions. Diaryl- and dialkyltellurium analogues appear more useful but less convenient. ${ }^{22,23}$

Some of the reported methods suffer from drawbacks like long reaction times, unsatisfactory yields, expensive reagents, two steps, and cumbersome experimental procedures. Therefore it seems highly desirable to find a simple, efficient, one step, and inexpensive protocol for 
synthesis of $\mathrm{N}$-sulfonylimines. We wish to report a simple protocol that makes these valuable building blocks readily available.

An important goal in green chemistry is represented by the elimination of volatile organic solvents, in fact solvent-free conditions (SFC) makes syntheses simpler, save energy, and prevent solvent waste, hazards, and toxicity. ${ }^{24}$ In addition the most important objectives now is to adapt classical processes so that pollution effects are kept to a minimum, with both a reduction in energy and consumption of raw materials. In this respect, dry-media reactions are promising, and a new approach has been undertaken using $\mathrm{AlCl}_{3}$ chemistry. As a part of our continued efforts to utilize the surface-mediated reaction(s) for preparation of N-sulfonylimines, here we wish to disclose a very simple, fast, general, highly efficient and improved one-step synthesis method for $\mathrm{N}$-sulfonylimine without any solvent in the presence of $\mathrm{AlCl}_{3}$ in relatively high yields.

\section{Results and Discussion}

In theory, the ideal procedure for obtaining $\mathrm{N}$-sulfonylimines would involve the condensation of carbonyl compounds with sulfonamides. However, the low nucleophilicity of the latter generally requires harsh acidic conditions to activate the carbonyl group, conditions that are usually incompatible with the resulting unstable sulfonylimines. ${ }^{25}$ In a program intended toward developing a cost-effective and direct method to this group of compounds in our laboratory, we exploit a method for preparation of new sulfonylimines 3a-3m. The reaction of the benzaldehyde with $p$-toluenesulfonamide was chosen as a model and its behavior was studied under a variety of conditions via TLC and NMR spectroscopy (Table 1). We found that the reaction of benzaldehyde and $p$-toluenesulfonamide did not proceed at room temperature without any catalyst/reagent even after 24 hrs (Table 1, entry 1).

Condensation of benzaldehyde with the $p$-toluenesulfonamide in appropriate solvent was carried out under reflux conditions (Table 1, entry 2). ${ }^{26}$ The final product was obtained in $2 \mathrm{hrs}$ of heating with low yields (15\%). Next, we screened $\mathrm{AlCl}_{3}$ under similar conditions. We found the yields were further improved to $30 \%$ (Table 1, entry 3 ).

In an attempt to introduce a green synthetic procedure and increase its rate and yield experiments were done under solvent-free conditions. The Lewis acids $\mathrm{SnCl}_{2}, \mathrm{CdCl}_{2}, \mathrm{CuCl}_{2}$, and $\mathrm{ZnCl}_{2}$ demonstrated a moderate ability for such reaction at $90{ }^{\circ} \mathrm{C}$ (Table 1 , entry $8,10,12$, and 14). The use of $\mathrm{FeCl}_{3}$ also exhibited a similar result at room temperature (Table 1, entry 15). When we compared the rate of product formation using $\mathrm{SnCl}_{2}, \mathrm{CdCl}_{2}, \mathrm{CuCl}_{2}, \mathrm{ZnCl}_{2}, \mathrm{FeCl}_{3}$ and $\mathrm{AlCl}_{3}$, it was seen that the $\mathrm{AlCl}_{3}$ was the more active. After only 15 minutes the yield of the product was $90 \%$ using $\mathrm{AlCl}_{3}$ (Table 1, entry 17). 
Table 1. Reaction of $p$-toluenesulfonamide $(1 \mathrm{mmol})$ with benzaldehyde $(1 \mathrm{mmol})$ under various conditions

\begin{tabular}{|c|c|c|c|}
\hline Entry & Conditions & Time(min) & Yield $^{\mathrm{a}} \%$ \\
\hline 1 & No catalyst/r.t. & 1440 & 0 \\
\hline 2 & Toluene/reflux ${ }^{26}$ & 120 & 15 \\
\hline 3 & $\mathrm{AlCl}_{3} /$ Toluene/reflux & 120 & 30 \\
\hline 4 & $\mathrm{Si}(\mathrm{OEt})_{4} / 160^{\circ} \mathrm{C}^{27}$ & 3600 & 68 \\
\hline 5 & $\mathrm{TiCl}_{4}(0.5 \mathrm{mmol}) / \mathrm{Et}_{3} \mathrm{~N}, \mathrm{CH}_{2} \mathrm{Cl}_{2} / 0^{\circ} \mathrm{C}^{18}$ & 36 & 58 \\
\hline 6 & Silica chloride $/ 120^{\circ} \mathrm{C}^{28}$ & 180 & 75 \\
\hline 7 & $\mathrm{SnCl}_{2} \cdot 2 \mathrm{H}_{2} \mathrm{O}(1 \mathrm{mmol}) /$ r.t. & 120 & 15 \\
\hline 8 & $\mathrm{SnCl}_{2} \cdot 2 \mathrm{H}_{2} \mathrm{O}(1 \mathrm{mmol}) / 90^{\circ} \mathrm{C}$ & 120 & 40 \\
\hline 9 & $\mathrm{CdCl}_{2}(1 \mathrm{mmol}) /$ r.t. & 120 & 18 \\
\hline 10 & $\mathrm{CdCl}_{2}(1 \mathrm{mmol}) / 90^{\circ} \mathrm{C}$ & 120 & 45 \\
\hline 11 & $\mathrm{CuCl}_{2}(1 \mathrm{mmol}) /$ r.t. & 120 & 10 \\
\hline 12 & $\mathrm{CuCl}_{2}(1 \mathrm{mmol}) / 90^{\circ} \mathrm{C}$ & 120 & 50 \\
\hline 13 & $\mathrm{ZnCl}_{2}(1 \mathrm{mmol}) /$ r.t. & 120 & 16 \\
\hline 14 & $\mathrm{ZnCl}_{2}(1 \mathrm{mmol}) / 90^{\circ} \mathrm{C}$ & 120 & 70 \\
\hline 15 & $\mathrm{FeCl}_{3}(1 \mathrm{mmol}) /$ r.t. & 120 & 50 \\
\hline 16 & $\mathrm{Al}_{2} \mathrm{O}_{3}($ acidic $)(1 \mathrm{mmol}) /$ r.t. & 120 & 15 \\
\hline 17 & $\mathrm{AlCl}_{3}(0.5 \mathrm{mmol}) / \mathrm{r.t}$. & 15 & 90 \\
\hline
\end{tabular}

aisolated yields.

During the course of our studies aimed at developing solvent-free procedures, ${ }^{28-32}$ we have now discovered that $\mathrm{AlCl}_{3}$ alone is a very efficient reagent for preparation of $\mathrm{N}$-sulfonylimines at room temperature in high yield, without any of the environmental disadvantages of using toxic solvents (Scheme 1). To the best of our knowledge there is no previous report for preparation of $\mathrm{N}$-sulfonylimines by $\mathrm{AlCl}_{3}$. In a typical experiment, $\mathrm{AlCl}_{3}(0.5 \mathrm{mmol})$, benzaldehyde $(1 \mathrm{mmol})$ and $p$-toluenesulfonamide $(1 \mathrm{mmol})$ were mixed thoroughly. The mixture was ground using a mortar and pestle until the reaction was completed (15 minutes). The solid mixture was removed through a Celite pad and washed with ethyl acetate $(20 \mathrm{~mL})$. The solvent was evaporated with a rotary evaporator and the solid product was dissolved in warm ethylacetate $(10 \mathrm{~mL})$, treated with n-hexane $(35-50 \mathrm{~mL}$ ), and allowed to stand at r.t. for 5-6 h. During this time crystals formed which were collected by filtration, washed with n-hexane, and dried.<smiles>[R]OS(=O)N[R]([R])=O</smiles>

\section{Scheme 1}


Table 2. Preparation of $\mathrm{N}$-sulfonylimines by using $\mathrm{AlCl}_{3}$

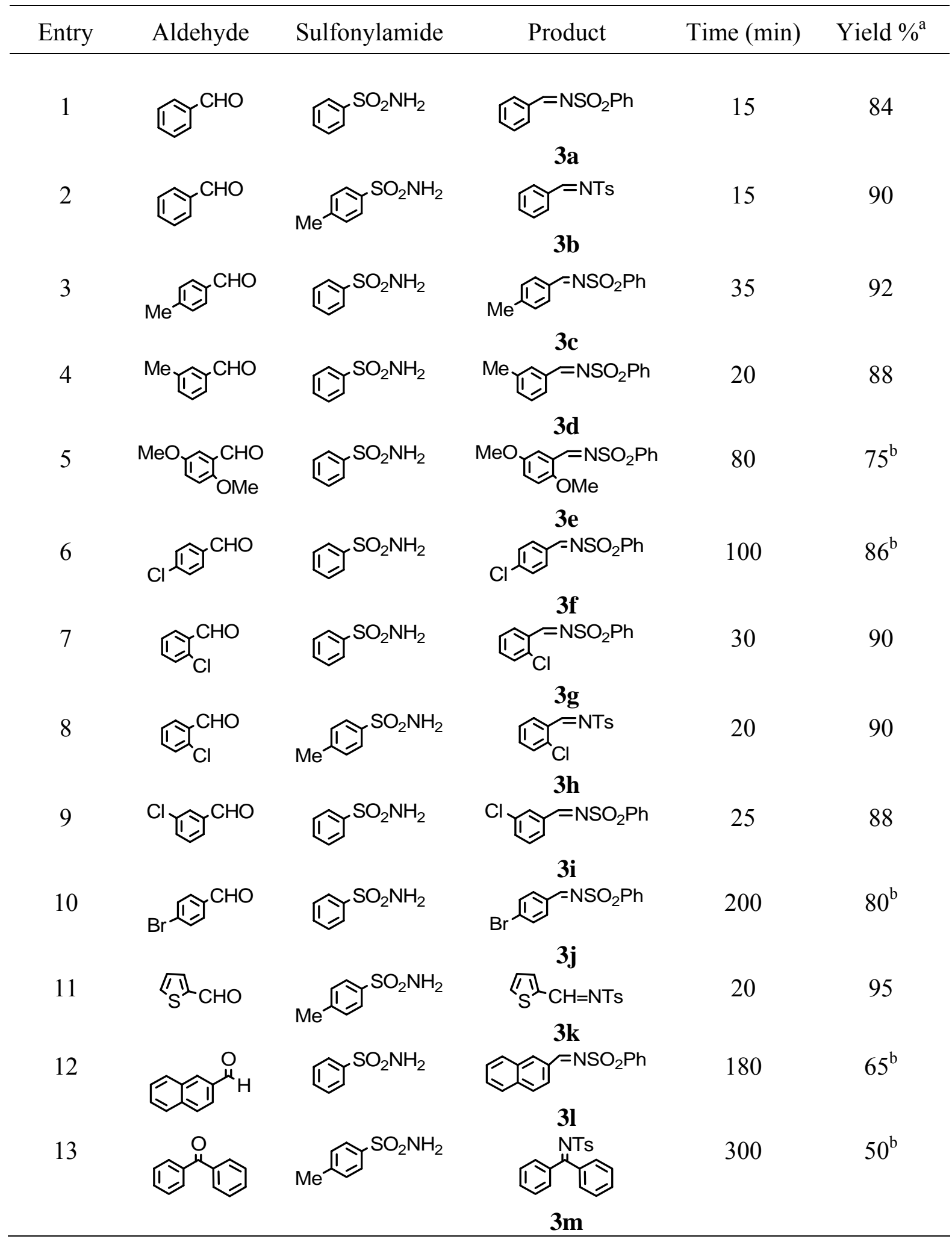

${ }^{a}$ Yields are the isolated compounds. ${ }^{b}$ The reaction was conducted in an oil bath at $85^{\circ} \mathrm{C}$. 
The generality and the scope of the reaction can be deduced from the results summarized in Table 2. We were pleased to find that a diverse range of aromatic aldehydes in the presence of $\mathrm{AlCl}_{3}$ were mixed with sulfonamides at room temperature, the corresponding $\mathrm{N}$-sulfonylimines were obtained in a high state of purity and in good yield.

According to Table 2, aromatic aldehydes containing both electron withdrawing and donating substituents (entries 2-8) afforded the corresponding N-sulfonyl aldimines in high yields. The Nsulfonylimines (3a-m) have $\mathrm{C}=\mathrm{N}$ bond stretching frequencies in the range of $1587-1606 \mathrm{~cm}^{-1}$. These compounds have a characteristic imino resonance in their ${ }^{1} \mathrm{H}$ NMR spectra at ca. $\delta$ 9-9.5. Mention must be made here that the conversion rate of $p$-toluenesulfonamide with aromatic aldehydes was a little higher than benzensulfonamide. This chemistry was applied unsuccessfully toward the synthesis of enolizable $\mathrm{N}$-sulfonyl aldimines and unfortunately this method shares a common limitation with several other methods of $N$-tosylaldimine synthesis ${ }^{18,33,34,36,37}$ in that it does not appear to be applicable to the synthesis of N-sulfonyl aldimines derived from aldol condensations being obtained instead. We have also studied the reaction of sulfonamide with ketones. In these reactions, the overall yields are not as high as those obtained from the aldehydes as a result of the lower efficiency of the condensation, which in the case of the benzophenone treated with $p$-toluenesulfonamide was only $50 \%$ after $5 \mathrm{~h}$, and acetophenone appears to be formed in admixture with other unidentified products. Methods of overcoming this limitation are currently under investigation.

The present one-step and high yielding protocol for preparation of $\mathrm{N}$-sulfonyl aldimines provides a better alternative to the existing methods due to its shorter reaction time, simple reaction procedure and the formation of cleaner products that can be used for synthetic applications without further purification.

\section{Experimental Section}

General Procedures. NMR spectra were recorded on a Bruker Avance DPX-250 $\left({ }^{1} \mathrm{H}\right.$ NMR 250 $\mathrm{MHz}$ and ${ }^{13} \mathrm{C}$ NMR $62.9 \mathrm{MHz}$ ) spectrometer in pure deuterated solvents with tetramethylsilane as an internal standard. Infrared spectra were obtained using a Shimadzu FT-IR 8300 spectrophotometer. Mass spectra were determined on a Shimadzu GCMS-QP 1000 EX instrument at 70 or $20 \mathrm{eV}$. Melting points were determined in open capillary tubes in a Buchi535 circulating oil melting point apparatus. Elemental analyses were obtained using a Thermofinnigan Flash-Ea 1112 series apparatus. The purity determination of the substrates and reaction monitoring were accomplished by TLC on silica gel PolyGram SILG/UV 254 plates. Column chromatography was carried out on short columns of silica gel 60 (70-230 mesh) in glass columns (2-3 cm diameter) using 15-30 gram of silica gel per one gram of crude mixture. Chemical materials were either prepared in our laboratories or were purchased from Fluka, Aldrich and Merck Companies. 
Preparative procedures. After a mortar was charged with anhydrous or hydrous $\mathrm{AlCl}_{3}(0.5$ mmol), sulfonamide $(1 \mathrm{mmol})$ and aldehyde $(1 \mathrm{mmol})$, the mixture was ground with a pestle at room temperature (reactions that were not complete $3 \mathrm{~h}$ at room temperature were repeated in a round bottom flask and heated in an oil bath at $85^{\circ} \mathrm{C}$ ) for certain period of time (Table 2). The solid mixture was removed through a Celite pad and washed with ethyl acetate $(20 \mathrm{~mL})$. The solvent was evaporated with a rotary evaporator and the solid product was dissolved in warm ethyl acetate $(10 \mathrm{~mL})$, treated with n-hexane $(35-50 \mathrm{~mL})$, and allowed to stand at r.t. for 5-6 h. During this time crystals formed which were collected by filtration, washed with n-hexane, and dried. In the case of benzophenone the pure product was obtained by chromatography on silica gel (ethyl acetate/ $n$-hexane as eluent).

$N$-[Phenylmethylidene]benzenesulfonamide (3a). White crystals in $84 \%$ yield. $\mathrm{Mp}=75-77{ }^{\circ} \mathrm{C}$ (lit. $\left.{ }^{18} 77-80{ }^{\circ} \mathrm{C}\right)$; IR (KBr): 1596, 1571, 1446, 1313, 1163, 1087, 800; ${ }^{1} \mathrm{H} \mathrm{NMR}\left(\mathrm{CDCl}_{3}, 250\right.$ $\mathrm{MHz}) \delta 7.41(\mathrm{t}, 2 \mathrm{H}, J=7.6 \mathrm{~Hz}), 7.47(\mathrm{t}, 2 \mathrm{H}, J=7.6 \mathrm{~Hz}), 7.52-7.56(\mathrm{~m}, 2 \mathrm{H}), 7.86(\mathrm{~d}, 2 \mathrm{H}, J=7.2 \mathrm{~Hz})$, 7.94(d, 2H, $J=7.1 \mathrm{~Hz}) ; 8.99(\mathrm{~s}, 1 \mathrm{H}) ;{ }^{13} \mathrm{C} \mathrm{NMR}\left(\mathrm{CDCl}_{3}, 62.9 \mathrm{MHz}\right) \delta 126.3,128.0,129.1,130.2$, 131.3, 132.7, 133.5, 135.0, 170.6; MS, m/z (\%), 245 (2.2, $\left.\mathrm{M}^{+}\right), 181$ (1.3), 141 (17.6), 104 (12.8), 77 (100), 51 (30.2). Anal. Calcd for $\mathrm{C}_{13} \mathrm{H}_{13} \mathrm{NO}_{2} \mathrm{~S}$ (245.298): Calc. C 63.65\%, H 4.52\%; found C $63.55 \%, \mathrm{H} 4.68 \%$.

4-Methyl- $N$-[phenylmethylidene]benzenesulfonamide(3b). White crystals in $90 \%$ yield. $\mathrm{Mp}=$ 107-108 ${ }^{\circ} \mathrm{C}$ (lit. ${ }^{18} 109-110{ }^{\circ} \mathrm{C}$ ); IR (KBr): 1606, 1593, 1450, 1319, 1159, 1089, 783; ${ }^{1} \mathrm{H}$ NMR $\left(\mathrm{CDCl}_{3}, 250 \mathrm{MHz}\right) \delta 2.32(\mathrm{~s}, 3 \mathrm{H}), 7.23(\mathrm{~d}, 2 \mathrm{H}, J=7.3 \mathrm{~Hz}), 7.37(\mathrm{~d}, 2 \mathrm{H}, J=7.3 \mathrm{~Hz}), 7.51(\mathrm{t}, 1 \mathrm{H}, J$ $=7.4 \mathrm{~Hz}), 7.77-7.83(\mathrm{~m}, 4 \mathrm{H}), 8.99(\mathrm{~s}, 1 \mathrm{H}) ;{ }^{13} \mathrm{C} \mathrm{NMR}\left(\mathrm{CDCl}_{3}, 62.9 \mathrm{MHz}\right) \delta 20.6,125.3,126.7$, 128.1, 128.7, 128.8, 132.5, 133.9, 143.6, 169.2; MS, m/z (\%), $259\left(1.7, \mathrm{M}^{+}\right), 195(0.9), 171$ (15.8), 155 (23.7), 107 (13.4), 91 (100), 65 (36.3). Anal. Calcd for $\mathrm{C}_{14} \mathrm{H}_{13} \mathrm{NO}_{2} \mathrm{~S}$ (259.321): Calc. C $64.84 \%$, H 5.05\%; found C $64.70 \%$, H 5.18\%

$N$-[(4-Methylphenyl)methylidene]benzenesulfonamide(3c). White crystals in 92\% yield. Mp = 114-115 ${ }^{\circ} \mathrm{C}$ (lit. ${ }^{33} 116-118{ }^{\circ} \mathrm{C}$ ); IR (KBr): 1594, 1562, 1448, 1311, 1160, 1089, 806, 756; ${ }^{1} \mathrm{H}$ $\operatorname{NMR}\left(\mathrm{CDCl}_{3}, 250 \mathrm{MHz}\right) \delta 2.36(\mathrm{~s}, 3 \mathrm{H}), 7.21(\mathrm{~d}, 2 \mathrm{H}, J=7.3 \mathrm{~Hz}), 7.47(\mathrm{t}, 2 \mathrm{H}, J=7.5 \mathrm{~Hz}), 7.50(\mathrm{t}$, $1 \mathrm{H}, J=7.6 \mathrm{~Hz}), 7.76(\mathrm{~d}, 2 \mathrm{H}, J=7.7 \mathrm{~Hz}), 7.93(\mathrm{~d}, 2 \mathrm{H}, J=8.1 \mathrm{~Hz}), 9.27(\mathrm{~s}, 1 \mathrm{H}) ;{ }^{13} \mathrm{C} \mathrm{NMR}\left(\mathrm{CDCl}_{3}\right.$, $62.9 \mathrm{MHz}) \delta 21.9,126.4,127.9,129.1,129.9,131.5,132.7,133.4,146.5,170.4 ; \mathrm{MS}, \mathrm{m} / \mathrm{z}(\%)$, 259 (1.0, M ), 195 (0.3), 171 (0.5), 141 (12.6), 77 (100), 51 (39.7). Anal. Calcd for $\mathrm{C}_{14} \mathrm{H}_{13} \mathrm{NO}_{2} \mathrm{~S}$ (259.321): Calc. C $64.84 \%$, H 5.05\%; found C $64.71 \%$, H 5.17\%

$\boldsymbol{N}$-[(3-Methylphenyl)methylidene]benzenesulfonamide(3d). White crystals in $88 \%$ yield. Mp $=85-88{ }^{\circ} \mathrm{C}$; IR (KBr):1596, 1575, 1448, 1313, 1162, 1092, 1004, 824; ${ }^{1} \mathrm{H} \mathrm{NMR}\left(\mathrm{CDCl}_{3}, 250\right.$ $\mathrm{MHz}) \delta 2.30(\mathrm{~s}, 3 \mathrm{H}), 7.28(\mathrm{~s}, 1 \mathrm{H}), 7.32(\mathrm{~d}, 1 \mathrm{H}, J=7.3 \mathrm{~Hz}), 7.37(\mathrm{~d}, 1 \mathrm{H}, J=7.4 \mathrm{~Hz}), 7.46(\mathrm{t}, 2 \mathrm{H}, J$ $=7.5 \mathrm{HZ}), 7.49(\mathrm{t}, 1 \mathrm{H}, J=7.6 \mathrm{~Hz}), 7.52(\mathrm{t}, 1 \mathrm{H}, J=7.6 \mathrm{~Hz}), 8.34(\mathrm{~d}, 2 \mathrm{H}, J=8.1 \mathrm{~Hz}), 9.27(1 \mathrm{H}, \mathrm{s}) ;{ }^{13} \mathrm{C}$ NMR $\left(\mathrm{CDCl}_{3}, 62.9 \mathrm{MHz}\right) \delta 21.1,126.3,129.0,129.1,131.4,132.2,132.6,133.5,136.0,138.2$, 139.1, 170.9; MS, m/z (\%), 259 (3.4, M+), 195 (1.6), 157 (5.8), 141 (17.1), 118 (20.8), 77 (100),

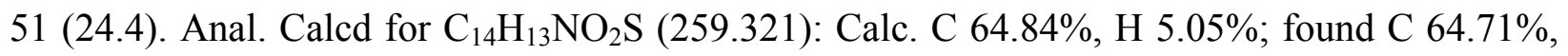
H $5.17 \%$ 
$N$-[(2,5-Dimethoxyphenyl)methylidene]benzenesulfonamide(3e). Light green crystals in 75\% yield. $\mathrm{Mp}=138-141{ }^{\circ} \mathrm{C}$; IR (KBr): $1587,1496,1321,1284,1162,1087,827 ;{ }^{1} \mathrm{H} \mathrm{NMR}\left(\mathrm{CDCl}_{3}\right.$, $250 \mathrm{MHz}) \delta 3.72(\mathrm{~s}, 3 \mathrm{H}), 3.77(\mathrm{~s}, 3 \mathrm{H}), 6.89(\mathrm{~d}, 1 \mathrm{H}, J=7.0 \mathrm{~Hz}), 7.16(\mathrm{~d}, 1 \mathrm{H}, J=7.4 \mathrm{~Hz}), 7.51(\mathrm{~s}$, $1 \mathrm{H}), 7.53-7.65(\mathrm{~m}, 3 \mathrm{H}), 8.00(\mathrm{~d}, 2 \mathrm{H}, J=8.1 \mathrm{~Hz}), 9.87(\mathrm{~s}, 1 \mathrm{H}),{ }^{13} \mathrm{C} \mathrm{NMR}\left(\mathrm{CDCl}_{3}, 62.9 \mathrm{MHz}\right) \delta$ 55.9, 56.1 , 110.8, 113.0, 125.2, 127.9, 129.0, 133.3, 153.5, 166.7; MS, m/z (\%), $305\left(19.0, \mathrm{M}^{+}\right)$, 163 (57.4), 149(41.7), 107(24.2), 77(100.0), 57 (82.0). Anal. Calcd for $\mathrm{C}_{15} \mathrm{H}_{15} \mathrm{NO}_{4} \mathrm{~S}$ (305.346): Calc. C $59.00 \%$, H $4.95 \%$; found C $59.20 \%$, H $4.81 \%$

$N$-[(4-Chlorophenyl)methylidene]benzenesulfonamide(3f). White crystals in $86 \%$ yield. Mp $=$ 131-133 ${ }^{\circ} \mathrm{C}$ (lit. ${ }^{33} 127-130{ }^{\circ} \mathrm{C}$ ); IR (KBr):1605, 1564, 1448, 1317, 1170, 1085, 800; ${ }^{1} \mathrm{H}$ NMR $\left(\mathrm{CDCl}_{3}, 250 \mathrm{MHz}\right) \delta 7.40(\mathrm{~d}, 2 \mathrm{H}, J=7.4 \mathrm{~Hz}), 7.49(\mathrm{t}, 2 \mathrm{H}, J=7.5 \mathrm{~Hz}), 7.58(\mathrm{t}, 1 \mathrm{H}, J=7.6 \mathrm{~Hz})$, 7.81(d, 2H, $J=7.7 \mathrm{~Hz}), 7.95(\mathrm{~d}, 2 \mathrm{H}, J=8.2 \mathrm{~Hz}), 9.02(\mathrm{~s}, 1 \mathrm{H}) ;{ }^{13} \mathrm{C} \mathrm{NMR}\left(\mathrm{CDCl}_{3}, 62.9 \mathrm{MHz}\right) \delta$ 124.0, 128.0, 129.2, 129.5, 129.6, 132.4, 133.6, 136.3, 169.0; MS, m/z (\%), $279\left(2.6, \mathrm{M}^{+}\right), 157$ (4.1), 141 (21.6), 77 (100.0), 51 (26.0). Anal. Calcd for $\mathrm{C}_{13} \mathrm{H}_{10} \mathrm{ClNO}_{2} \mathrm{~S}$ (279.740): Calc. C $55.82 \%$, H 3.60\%; found C 55.69\%, H 3.49\%

$N$-[(2-Chlorophenyl)methylidene]benzenesulfonamide(3g). White crystals in $90 \%$ yield. Mp $=$ 117-119 ${ }^{\circ} \mathrm{C}$; IR (KBr):1587, 1561, 1447, 1324, 1159, 1088, 802, 690; ${ }^{1} \mathrm{H} \mathrm{NMR}\left(\mathrm{CDCl}_{3}, 250\right.$ $\mathrm{MHz}) \delta 7.25(\mathrm{t}, 1 \mathrm{H}, J=7.6 \mathrm{~Hz}), 7.39(\mathrm{t}, 2 \mathrm{H}, J=7.5 \mathrm{~Hz}), 7.48(\mathrm{t}, 1 \mathrm{H}, J=7.0 \mathrm{~Hz}), 7.50(\mathrm{~d}, 1 \mathrm{H}, J=7.5$ $\mathrm{Hz}), 7.57(\mathrm{t}, 1 \mathrm{H}, J=7.5 \mathrm{~Hz}), 7.94(\mathrm{~d}, 2 \mathrm{H}, J=8.3 \mathrm{~Hz}), 8.06(\mathrm{~d}, 1 \mathrm{H}, J=7.3 \mathrm{~Hz}), 9.43(\mathrm{~s}, 1 \mathrm{H}) ;{ }^{13} \mathrm{C}$ NMR $\left(\mathrm{CDCl}_{3}, 62.9 \mathrm{MHz}\right) \delta 127.4,128.1,129.2,129.6,130.1,130.5,133.7,135.7,137.7,139.0$, 167.2, MS, m/z (\%), $279\left(0.9, \mathrm{M}^{+}\right), 244$ (23.1), 141 (17.6), 77 (100.0), 51 (21,8). Anal. Calcd for $\mathrm{C}_{13} \mathrm{H}_{10} \mathrm{ClNO}_{2} \mathrm{~S}$ (279.740): Calc. C 55.82\%, H 3.60\%; found C 55.70\%, H 3.76\%

$\mathrm{N}$-[(2-Chlorophenyl)methylidene]-4-methylbenzenesulfonamide(3h). White crystals in $90 \%$ yield. $\mathrm{Mp}=121-123{ }^{\circ} \mathrm{C}$ (lit. $\left.{ }^{34} 128-129{ }^{\circ} \mathrm{C}\right)$; IR (KBr):1587, 1580, 1434, 1320, 1157, 1087, 789, 707, 669; ${ }^{1} \mathrm{H}$ NMR $\left(\mathrm{CDCl}_{3}, 250 \mathrm{MHz}\right) \delta 2.35(\mathrm{~s}, 3 \mathrm{H}), 7.29(\mathrm{~d}, 2 \mathrm{H}, J=8.2), 7.37-7.47(\mathrm{~m}, 2 \mathrm{H})$, 7.74(d, $1 \mathrm{H}, J=8.3 \mathrm{~Hz}), 8.33(\mathrm{~d}, 2 \mathrm{H}, J=8.3 \mathrm{~Hz}), 8.08(\mathrm{~d}, 1 \mathrm{H}, J=7.9 \mathrm{~Hz}), 9.42(\mathrm{~s}, 1 \mathrm{H}) ;{ }^{13} \mathrm{C}$ NMR $\left(\mathrm{CDCl}_{3}, 62.9 \mathrm{MHz}\right) \delta 21.6,126.4,127.3,128.2,129.6,129.8,130.1,130.4,135.6,138.9,144.8$, 166.7; MS, m/z (\%), 293 (0.9, $\mathrm{M}^{+}$), 258 (2.7), 183 (0.7), 155 (21.8), 91 (100.0), 57 (37.5). Anal. Calcd for $\mathrm{C}_{13} \mathrm{H}_{10} \mathrm{ClNO}_{2} \mathrm{~S}$ (293.028): Calc. C 55.82\%, H 3.60\%; found C 55.68\%, H 3.79\%

$N$-[(3-Chlorophenyl)methylidene]benzenesulfonamide(3i). White crystals in $88 \%$ yield. $\mathrm{Mp}=$ 92-94 ${ }^{\circ} \mathrm{C}$; IR (KBr):1606, 1565, 1446, 1326, 1167, 1086, 794; ${ }^{1} \mathrm{H}$ NMR $\left(\mathrm{CDCl}_{3}, 250 \mathrm{MHz}\right) \delta$ 7.44(t, $1 \mathrm{H}, J=7.8 \mathrm{~Hz}), 7.52-7.59(3 \mathrm{H}, \mathrm{m}), 7.57(\mathrm{~s}, 1 \mathrm{H}), 7.65(\mathrm{~d}, 1 \mathrm{H}, J=7.1 \mathrm{~Hz}), 7.79(\mathrm{~d}, 1 \mathrm{H}, J=7.6$ $\mathrm{Hz}), 8.01(\mathrm{~d}, 2 \mathrm{H}, J=7.1 \mathrm{~Hz}), 9.01(\mathrm{~s} .1 \mathrm{H}) ;{ }^{13} \mathrm{C} \mathrm{NMR}\left(\mathrm{CDCl}_{3}, 62.9 \mathrm{MHz}\right) \delta 125.9,127.6,128.6$, 128.7, 129.4, 129.8, 129.9, 132.3, 133.3, 134.3, 168.5; MS, m/z (\%), $279\left(0.9, \mathrm{M}^{+}\right), 171(1.0)$, 141 (18.3), 94 (12.5), 77 (100.0), 51 (47.5). Anal. Calcd for $\mathrm{C}_{13} \mathrm{H}_{10} \mathrm{ClNO}_{2} \mathrm{~S}$ (279.740): Calc. C $55.82 \%$, H 3.60\%; found C 55.60\%, H 3.75\%

$\boldsymbol{N}$-[(4-Bromophenyl)methylidene]benzenesulfonamide(3j). White crystals in $80 \%$ yield. $\mathrm{Mp}=$ 194-195 ${ }^{\circ} \mathrm{C}$; IR (KBr):1606, 1560, 1448, 1319, 1164, 1089, 796, 663, 557; ${ }^{1} \mathrm{H} \mathrm{NMR}\left(\mathrm{CDCl}_{3}, 250\right.$ $\mathrm{MHz}) \delta 7.40(\mathrm{~d}, 2 \mathrm{H}, J=8.5 \mathrm{~Hz}), 7.49(\mathrm{t}, 2 \mathrm{H}, J=7.7 \mathrm{~Hz}), 7.58(\mathrm{t}, 1 \mathrm{H}, J=7.4 \mathrm{~Hz}), 7.81(\mathrm{~d}, 2 \mathrm{H}, J=8.8$ $\mathrm{Hz}), 7.95(\mathrm{~d}, 2 \mathrm{H}, J=8.0 \mathrm{~Hz}), 9.20(\mathrm{~s}, 1 \mathrm{H}) ;{ }^{13} \mathrm{C} \mathrm{NMR}\left(\mathrm{CDCl}_{3}, 62.9 \mathrm{MHz}\right) \delta 124.0,127.2,, 128.0$, 129.2, 129.5, 133.5, 136.3, 170.54; MS, m/z (\%), 324 (0.1, $\left.\mathrm{M}^{+}\right), 215$ (1.6), 182 (1.7), 149 (4.9), 
77 (100.0), 51 (32.0). Anal. Calcd for $\mathrm{C}_{13} \mathrm{H}_{10} \mathrm{BrNO}_{2} \mathrm{~S}$ (324.194): Calc. C 48.16\%, H 3.11\%; found C $48.01 \%$, H $3.28 \%$

4-Methyl- $\mathrm{N}$-[2-thienylmethylidene]benzenesulfonamide(3k). White crystals in $95 \%$ yield. Mp $=104-105{ }^{\circ} \mathrm{C}$ (lit. $\left.{ }^{28} 98-103{ }^{\circ} \mathrm{C}\right)$; IR (KBr):1589, 1417, 1299, 1155, 819; ${ }^{1} \mathrm{H}$ NMR $\left(\mathrm{CDCl}_{3}, 250\right.$ MHz) $\delta 2.34(\mathrm{~s}, 3 \mathrm{H}), 7.25(\mathrm{~d}, 2 \mathrm{H}, J=7.3 \mathrm{~Hz}), 7.70(\mathrm{~d}, 2 \mathrm{H}, J=7.5 \mathrm{~Hz}), 7.74-7.80(\mathrm{~m}, 3 \mathrm{H}), 9.03(\mathrm{~s}$, $1 \mathrm{H}) ;{ }^{13} \mathrm{C} \mathrm{NMR}\left(\mathrm{CDCl}_{3}, 62.9 \mathrm{MHz}\right) \delta 21.6,126.4,127.9,128.8,129.6,129.7,136.7,139.1$, 144.4, 162.3; MS, m/z (\%), 265 (0.7, $\left.\mathrm{M}^{+}\right), 155$ (20.3), 107 (19.4), 91 (100), 65 (38.8). Anal. Calcd for $\mathrm{C}_{12} \mathrm{H}_{11} \mathrm{NO}_{2} \mathrm{~S}_{2}$ (265.353): Calc. C $54.32 \%, \mathrm{H} 4.18 \%$; found C $54.17 \%, \mathrm{H} 4.30 \%$

$N$-[2-Naphthylmethylidene]benzenesulfonamide(3l). Yellow crystals in $65 \%$ yield. $\mathrm{Mp}=116$ $117^{\circ} \mathrm{C}$ (lit. ${ }^{35} 172-174{ }^{\circ} \mathrm{C}$ ); IR (KBr): 1587, 1568, 1448, 1321, 1162, 1091,835; ${ }^{1} \mathrm{H}$ NMR $\left(\mathrm{CDCl}_{3}\right.$, $250 \mathrm{MHz}) \delta 7.46-7.60(\mathrm{~m}, 5 \mathrm{H}), 7.79-7.94(\mathrm{~m}, 4 \mathrm{H}), 7.98(\mathrm{~d}, 2 \mathrm{H}, J=8.0 \mathrm{~Hz}), 8.27(\mathrm{~s}, 1 \mathrm{H}), 9.13(\mathrm{~s}$, $1 \mathrm{H}) ;{ }^{13} \mathrm{C} \mathrm{NMR}\left(\mathrm{CDCl}_{3}, 62.9 \mathrm{MHz}\right) \delta 124.0,127.2,128.0,128.7,129.1,129.2,129.5,133.5$, 136.3, 170.5; MS, m/z (\%), 295 (2.5, M+), 185 (1.9), 157 (14.2), 94 (19.9), 77 (100), 51 (50.6). Anal. Calcd for $\mathrm{C}_{17} \mathrm{H}_{13} \mathrm{NO}_{2} \mathrm{~S}$ (295.357): Calc. C 69.13\%, H 4.44\%; found C 69.00\%, H 4.62\%

$\mathrm{N}$-(Diphenylmethylene)-4-methylbenzenesulfonamide(3m). Light yellow crystals in 50\% yield. $\mathrm{Mp}=103-105^{\circ} \mathrm{C}\left(\right.$ lit. $\left.^{36} 103{ }^{\circ} \mathrm{C}\right)$; IR $(\mathrm{KBr}): 1560,1317,1155,1089,829 ;{ }^{1} \mathrm{H} \mathrm{NMR}\left(\mathrm{CDCl}_{3}\right.$, $250 \mathrm{MHz}) \delta 2.33(\mathrm{~s}, 3 \mathrm{H}), 7.74(\mathrm{~d}, 2 \mathrm{H}, J=8.2 \mathrm{~Hz}), 7.47-7.42(\mathrm{~m}, 6 \mathrm{H}), 7.24-7.30(\mathrm{~m}, 4 \mathrm{H}), 7.19(\mathrm{~d}$, $2 \mathrm{H}, J=8.0 \mathrm{~Hz}) ;{ }^{13} \mathrm{C} \mathrm{NMR}\left(\mathrm{CDCl}_{3}, 62.9 \mathrm{MHz}\right) \delta 21.5,127.3,128.1,129.4,130.1,138.5,143.3$, 178.7; MS, m/z (\%), $335\left(0.2, \mathrm{M}^{+}\right), 271$ (0.6), 199 (0.7), 155 (21.1), 108 (22.4), 91 (100), 65 (41.1). Anal. Calcd for $\mathrm{C}_{20} \mathrm{H}_{17} \mathrm{NO}_{2} \mathrm{~S}$ (335.241): Calc. C 71.62\%, H 5.11\%; found C 71.48\%, H $5.22 \%$

\section{Acknowledgements}

We gratefully acknowledge the support of this work by the Shiraz University Research Council.

\section{References and Notes}

1. (a) Weinreb, S. M. Top. Curr. Chem. 1997, 190, 131. (b) Bloch, R. Chem. Rev. 1998, 98, 407.

2. (a) Melnick, M. D.; Freyer, A. J.; Weinreb, S. M. Tetrahedron Lett. 1989, 29, 3891. (b) Sisko, J.; Ralbovsky, J. L.; Kinsella, M. A.; Sisko, J.; Weinreb, S. M. Synth. Commun. 1990, 20, 573. (c) Alexander, M. D.; Anderson, R. E.; Sisko, J.; Weinreb, S. M. J. Org. Chem. 1990, 55, 2563. (d) Saigo, K.; Shimada, S.; Hasegawa, H. Chem. Lett. 1990, 905. (e) Reetz, M. T.; Jaeger, R.; Drewlies, R.; Hubel, M. Angew. Chem., Znt. Ed. Engl. 1991, 30, 103.

3. Albrecht, R.; Kresze, G. Chem. Ber. 1964, 97, 490.

4. Boger, D. L.; Weinreb, S. N. Hetero Diels-Alder Methodology in Organic Synthesis;Academic: San Diego, 1987. 
5. Boger, D. L.; Kasper, A. M. J. Am. Chem. Soc. 1989, 111, 1517.

6. Boger, D. L.; Corbett, W. L.; Curran, T. T.; Kasper, A. M. J. Am. Chem. Soc. 1991, 113, 1713.

7. Sisko, J.; Weinreb, S. M. Tetrahedron Lett. 1989, 30, 3037.

8. For an asymmetric version, see: McFarlane, A. K.; Thomas, G.; Whiting, A. Tetrahedron Lett. 1993, 34, 2379.

9. Tschaen, D. M.; Turos, E.; Weinreb, S. M. J. Org. Chem. 1984, 49, 5058.

10. Melnick, M. J.; Freyer, A. J.; Weinreb, S. M. Tetrahedron Lett. 1988, 29, 3891.

11. Sisko, J.; Weinreb, S. M. J. Org. Chem. 1990, 55, 393.

12. Yamada, K.; Fujihara, H.; Yamamoto, Y.; Miwa, Y.; Taba, T.; Tomioka, K. Org. Lett. 2002, 4, 3509.

13. Vass, A.; Dudas, J.; Varma, R. S. Tetrahedron Lett. 1999, 40, 4951.

14. Hori, R.; Aoayama, T.; Shoiri, T. Tetrahedron Lett. 2000, 41, 9455.

15. Arini, L. G.; Sinclair, A.; Szeto, P.; Stockan, R. A. Tetrahedron Lett. 2004, 45, 1589.

16. Davis, F. A.; Reddy, R . T.; Reddy, R . E. J. Org . Chem. 1992, 57, 6387.

17. Lichtenburger, J.; Fleury, S.; Barret, B. Bull. Soc. Chim. Fr. 1955, 669.

18. Jennings, W. B.; Lovely, C. J. Tetrahedron 1991, 47, 5561.

19. Albrecht, R.; Kresze, G.; Mlakar, B. Chem. Ber. 1964, 87, 487.

20. Glass, R.; Hoy, R. Tetrahedron. Lett. 1976, 21, 1781.

21. Derkach, N. Y.; Barashenkov, G. G.; Slyosarenko, E. Zh. Org. Khim. 1982, 18, 170.

22. Naddaka, V. I.; Garkin, V. P.; Obayan, K. V. Zh. Org. Khim. 1981, 17, 2020.

23. Suzuki, H.; Takeda, S.; Hanazaki, Y. Chem. Lett. 1985, 679.

24. Tanka, K.; Solvent Free Organic Synthesis, Wiley-VCH, Weinhein, (2003).

25. Liu, G.; Cogan, D. A.; Owens, T. D.; Tang, T. P.; Ellman, J. A. J. Org. Chem. 1999, 64, 1278 .

26. Wynne, J. H.; Price, E.; Rorer, J. R.; Stalick, W. M. Synth. Commun. 2003, 33, 341.

27. Love, B. E.; Raje, P. S.; Williams, T. C. Synlett 1994, 493.

28. Hasaninejad, A.; Sharghi, H. Phosphorus, Sulfur and Silicon and the Related Elements 2007, $182,873$.

29. Sharghi, H.; Hosseini Sarvari, M. J. Chem. Res. (S) 2000, 24.

30. (a) Sharghi, H.; Hosseini Sarvari, M. Synthesis 2003, 243. (b) Sharghi, H.; Hosseini Sarvari, M. Synthesis 2004, 2165. (c) Sharghi, H.; Shahsavari-Fard, Z. Helv. Chim. Acta 2005, 88, 42. (d) Sharghi, H.; Hosseini Sarvari, M. Helv. Chim. Acta 2005, 88, 2282. (e) Sharghi, H.; Hosseini Sarvari, M.; Eskandari, R. Synthesis 2006, 2047.

31. (a) Sharghi, H.; Kaboudin, B. J. Chem. Res. (S) 1998, 628. (b) Sharghi, H.; Niknam, K. Iran. J. Chem. \& Chem. Eng. 1999, 18, 36; Chem. Abstr. 2000, 132, 122357m. (c) Sharghi, H.; Hosseini Sarvari, M. J. Chem. Res. (S) 2001, 446. (d) Sharghi, H.; Hosseini Sarvari, M. J. Org. Chem. 2003, 68, 4096. (e) Sharghi, H.; Hosseini Sarvari, M. Synth. Commun. 2003, 33(2), 205. (f) Sharghi, H.; Hosseini Sarvari, M. Tetrahedron 2003, 59, 3627. (g) Sharghi, H.; Hosseini Sarvari, M. Synthesis 2003, 879. (h) Sharghi, H., Shahsavari-Fard, Z. J. Iranian 
Chem. Soc. 2005, 2, 47 (www.ics-ir.org/jics/). (i) Sharghi, H., Shahsavari-Fard, Z. Phosphorus, Sulfur, and Silicon 2005, 180, 1.

32. (a) Sharghi, H.; Niknam K.; Massah, A. R. J. Heterocycl. Chem. 1999, 36, 601. (b) Sharghi, H.; Hosseini Sarvari, M. Synlett 2001, 99. (c) Sharghi, H.; Hosseini Sarvari, M. Synthesis 2002, 1057. (d) Sharghi, H.; Hosseini Sarvari, M. Tetrahedron 2002, 58, 10323. (e) Sharghi, H.; Hosseini Sarvari, M. J. Chem. Res. (S) 2001, 446. (f) Sharghi, H.; Hosseini Sarvari, M. J. Chem. Res. (S) 2003, 176. (g) Kazemi, F.; Sharghi, H.; Nasseri, M. A. Synthesis 2004, 205. (h) Sharghi, H.; Hosseini Sarvari, M. J. Iranian Chem. Soc. 2004, 1(1), 28 (www.ics-ir.org/jics/). (i) Sharghi, H.; Hosseini Sarvari, M. J. Org. Chem. 2004, 69, 6953. (j) Sharghi, H.; Hosseini Sarvari, M.; Eskandari, R. J. Chem. Res. (S) 2005, 482. (k) Sharghi, H.; Hosseini Sarvari, M. Tetrahedron 2005, 61, 10903. (1) Sharghi, H.; Hosseini Sarvari, M. J. Chem. Res. (S) 2006, 205.

33. Jin, T.; Feng, G.; Yang, M.; Li, T. Synth. Comm. 2004, 34, 1277.

34. Trost, B. M.; Marrs, C. J. Org. Chem. 1991, 56, 6468.

35. Ruano, J. L. G.; Aleman, J.; Belen Cid, M.; Perra, A. Org. Lett. 2005, 7, 179.

36. Georg, G. I.; Harriman, G. C. B.; Peterson, S. A. J. Org. Chem. 1995, 60, 7366.

37. Chelma, F.; Hebbe, V; Normant, J. F. Synthesis 2000, 1, 75. 